\title{
Los medios sociales en el ejercicio del periodismo
}

\author{
Recibido: 01 de agosto de 2013 \\ Aceptado: 17 de marzo de 2014 \\ Publicado: 28 de noviembre de 2014
}

\author{
Amaro La Rosa \\ amaro@unife.edu.pe
}

Universidad Femenina del Sagrado Corazón (Perú)

\begin{abstract}
Resumen: Se presenta una breve visión panorámica de la influencia de los medios sociales en el ejercicio de la profesión periodística. Se describe el uso de los medios sociales como fuentes de información así como el impacto producido en el procesamiento de la información y en las rutinas de producción de la información noticiosa. Se concluye que los medios sociales influyen de manera creciente en la actividad periodística que se hace efectiva en la sociedad contemporánea.
\end{abstract}

Palabras clave: Medios sociales, periodismo, Facebook, Twitter, newsmaking, audiencia.

Abstract: The article presents one panoramic viewpoint on social media influence in the work of professional journalism. The use of social media as information sources and their impact on information processing and news production routines are described. It is concluded that social media influence growing in the journalistic activity that becomes effective in contemporary society.

Key words: Social media, Journalism, Facebook, Twitter, Newsmaking, Audience.

\section{Introducción}

Estamos convencidos que a lo largo de la historia de los medios de comunicación se registra una relación constante y de raíces muy profundas entre comunicación, tecnología y sociedad. El dinamismo es un rasgo distintivo de la sociedad y es indudable que en las últimas décadas del siglo XX y en lo que va del XXI, su velocidad relativa ha sido comparativamente mayor en comparación con otras épocas de la historia. 
La tecnología, entendida como la aplicación de soluciones creativas a los problemas cotidianos del ser humano igualmente ha evolucionado ostensiblemente. En consonancia con estos dos elementos se hace patente el desarrollo de la comunicación masiva en sus diversas manifestaciones y que, para decirlo con un lenguaje tecnológico actualizado, se "vehiculizan" a través de distintas plataformas. La rápida obsolescencia de los productos tecnológicos utilizados para comunicarnos es una clara muestra de cómo estos avances se hacen efectivos en la sociedad de la información.

En este contexto es evidente, apelando a una visión posmodernista, que el cambio es una constante, que la profesión periodística ha experimentado modificaciones muy aceleradas en los últimos años. Cambios en los recursos tecnológicos que eran inimaginables, configuran ahora elementos indispensables para el trabajo profesional cotidiano, que han determinado notables transformaciones en los tiempos, el procesamiento de datos y las rutinas de trabajo del periodista así como una sensible variación en la vinculación con los usuarios, quienes en el mundo contemporáneo tienen la posibilidad de ser cada vez más activos y de generar sus propios contenidos.

No obstante, cuando se compuso el presente artículo se evita encasillar la labor periodística en el interior de los medios de comunicación tradicionales, en tanto el trabajo profesional se ejerce actualmente en múltiples áreas, unas ya plenamente consolidadas, otras que se encuentran aún en estado incipiente, mientras que algunas (usando una expresión del argot de Internet) podría decirse que están en pleno proceso de construcción. Es precisamente en estas últimas en las cuales tienen una marcada injerencia recursos tecnológicos, tales como los que analizamos a lo largo del artículo.

Las redes sociales existen desde los albores de la humanidad, como expresión evidente de lo que se ha tendido en denominar el instinto gregario del ser humano, el cual presumiblemente involucraba en sus inicios la unión para la supervivencia en un ambiente hostil, para luego facilitar el desarrollo de diversas modalidades de comunicación y con ellas el surgimiento y consolidación de las culturas. A lo largo de la historia estos nexos cotidianos entre los seres humanos han adquirido paulatinamente una configuración propia dependiente de los contextos socioculturales en las cuales funcionaron y de los cambios que se produjeron en los mismos. A medida que la sociedad evolucionaba, naturalmente sucedía lo mismo con las redes sociales que funcionaban en su seno.

Para los especialistas (pero también para el acucioso integrante de la audiencia) no constituye un secreto que Internet ha condicionado un considerable impacto sobre los ahora denominados medios tradicionales. Es indudable que Internet ha viabilizado el logro de modalidades de interacción previamente inexistentes así como ha producido profundas modificaciones en otras preexistentes. Las cifras globales de su crecimiento registradas por la Unión Internacional de Telecomunicaciones (ITU, 2013) son reveladoras: 2,700 millones de personas, vale decir alrededor del $40 \%$ de la población mundial está conectado a Internet y el número de teléfonos móviles (6,800 millones) se acerca al de la población del planeta. 
En este marco, los medios sociales que se desarrollaron con la web 2.0, realmente han revolucionado la comunicación cotidiana entre los seres humanos, que ahora no pueden vivir sin estar conectados, pero también han impactado en la comunicación masiva en general y en el ámbito periodístico en particular, tal como apreciaremos en las siguientes páginas. Como uno de sus recursos más populares, los medios sociales vinieron para quedarse y para transformar nuestro trabajo con herramientas que inicialmente se consideramos irrelevantes, pero cuya presencia forma ahora parte de la actividad cotidiana de los medios.

¿Quién iba a decirnos hace algunos años que 140 palabras colocadas en el ciberespacio por algún personaje público, pasarían a ocupar lugar central en los informativos nocturnos y posteriormente rebotarían en la mayoría de las primeras planas de los matutinos del día siguiente, logrando en ciertos casos un enorme impacto sobre la opinión pública? ¿Quién iba a predecir que el uso intensivo de mensajes en redes sociales sería uno de los factores que hicieron posible el derrocamiento de regímenes dictatoriales que parecían inamovibles; tal como ocurrió con la primavera árabe? ¿Algún director de noticias de televisión podía imaginarse años atrás que estaría en condiciones de recibir por escrito el feedback inmediato de su audiencia ante cierta información y que podría presentarlo inmediatamente después en la pantalla?

Enfocándonos en los usuarios, podemos hablar en la actualidad del consumo multiplataforma pues amén de la PC, es cada vez más frecuente el empleo de la laptop, del teléfono móvil con acceso a Internet y de manera creciente de las tablets y phablets, que facilitan las posibilidades de interacción así como un incremento en la calidad de imagen y sonido. Al respecto, según un estudio de Duggan y Smith (2013), el $42 \%$ de los adultos utilizan múltiples plataformas de red social y para quienes usan solamente un sitio, habitualmente el elegido es Facebook.

\section{Algunas consideraciones sobre los medios sociales}

Existe una diversidad de enfoques conceptuales sobre la naturaleza de los medios sociales. Sobre este particular, no intentaremos realizar precisiones epistemológicas, solamente consignaremos algunos criterios que nos parecen relevantes para nuestro trabajo. García Govaze (2011: 33) define como medios sociales a "todas aquellas herramientas tecnológicas que permiten una comunicación digital social entre individuos".

Desde lo que entendemos como una visión sistémica, Cabañas (2011: 20) concibe que los medios sociales constituyen "estructuras sociales que se puede representar en forma de uno o varios grafos en los cuales los nodos representan individuos, a veces de-nominados actores, y las aristas relaciones entre ellos". Constituyen para Boyd y Ellison (2007) servicios web que posibilitan al usuario:

- Construir un perfil.

- Articular una lista de otros usuarios.

- Visualizar su lista de contactos. 
Para Boyd (2011: 259), son de manera simultánea:

- El espacio construido a través de la tecnología de redes.

- El colectivo imaginado que surge como resultado de la intersección de las personas, la tecnología y la práctica.

Por nuestra parte, estimamos que los medios sociales son plataformas web que brindan una serie de servicios interactivos y de creación de contenidos a sus usuarios registrados quienes tienen diversas modalidades de contacto en red con características preestablecidas.

Cuando hablamos de medios sociales no nos estamos refiriendo por cierto a un todo homogéneo, sino a un conglomerado de recursos ubicables en el ciberespacio, con características diferenciadas, que sirven a grupos distintos de diversas maneras, pero que tienen algo en común: la "dialogicidad", que supone la posibilidad que el perceptor pueda desempeñarse como emisor, convirtiendo en realidad el criterio conceptual de "prosumidores", que acuñara Alvin Toffler décadas atrás. El mapa que reproducimos a continuación (figura 1) constituye una visión panorámica de los diversos recursos y de la naturaleza del contacto que involucran los medios sociales.

Figura 1: Contactos y herramientas de comunicación.

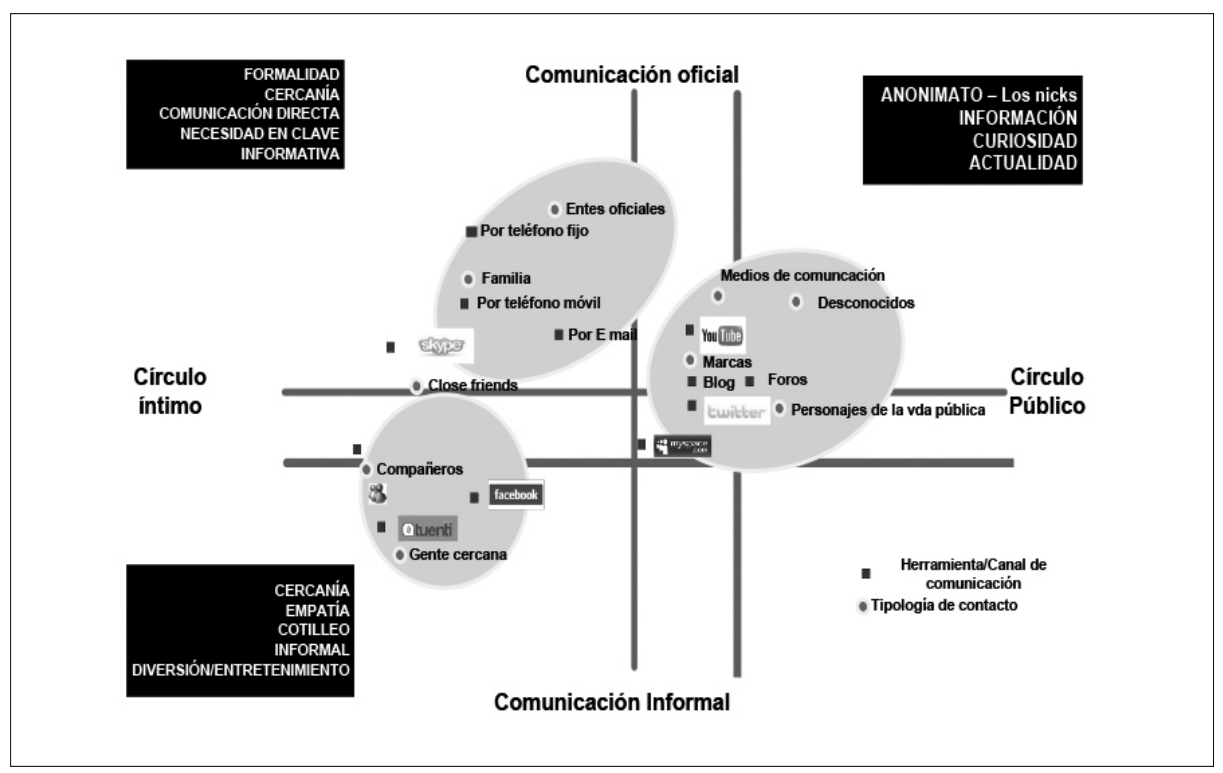

Fuente: The cocktail analysis (2011).

1. Bruns (2013) prefiere denominarlos "produsuarios" en tanto sus prácticas no involucran la tradicional producción de contenidos sino que van más allá al contribuir al mejoramiento de los mismos mediante su construcción y ampliación. 
Los medios sociales figuran entre los más populares recursos de Internet a nivel mundial. A inicios del 2014, Facebook contaba con 1310 millones de usuarios y Twitter más de 645 millones. Tal como lo especificamos en la figura 2, para mediados de abril del 2014, varios medios sociales se ubicaban entre los 10 sitios más populares del mundo:

Figura 2: Sitios web favoritos en el mundo.

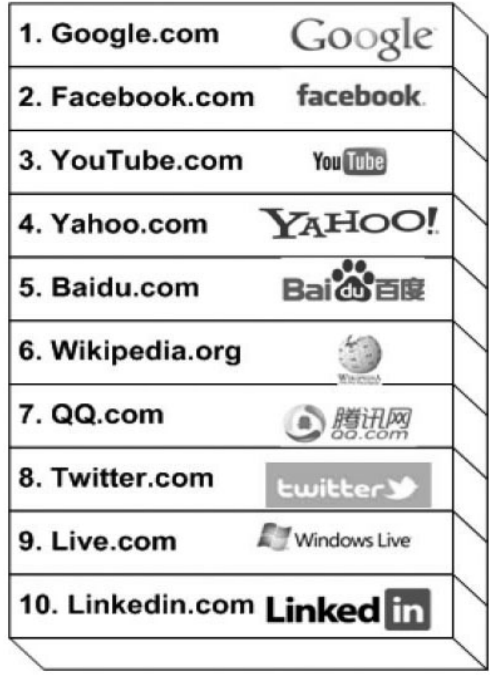

Fuente: Elaboración propia, basada en Alexa (2014).

Lo anterior expresa el incremento en el uso de medios sociales y su creciente importancia en el mundo de hoy, que en los países desarrollados se relaciona a nuestro entender con las siguientes variables:

- $\quad$ El crecimiento del mercado de Internet.

- $\quad$ El auge de la banda ancha que posibilita acceso más rápido, con mayor volumen de datos y alta calidad.

- La difusión del servicio de Wi-fi en ciudades y locales públicos: Facilita el acceso a Internet usando laptops y tablets.

- El mayor consumo de teléfonos inteligentes con conexión a Internet y a las principales redes sociales.

- El incremento en el consumo de tablets.

El aumento de usuarios de medios sociales en los países en desarrollo no sigue el mismo ritmo en todo el mundo. En el caso del Perú, que figura entre los países con mayor promedio de horas de consumo, se debe a los siguientes factores: 
- $\quad$ El uso de cabinas públicas: Desde la década del noventa el Perú fue pionero en este sistema de acceso a Internet que ha permitido sobrepasar la denominada brecha digital a millones de personas en todo el país, a un costo accesible.

- El aumento de líneas dedicadas en los hogares: La disminución de costos del servicio de los proveedores de Internet ha favorecido el acceso doméstico, lo cual implica tanto un significativo ahorro como la posibilidad de disponer del servicio sin restricciones las 24 horas del día.

- El mayor consumo de teléfonos móviles inteligentes. La economía de escala y la rápida obsolescencia de la tecnología hace factible actualmente la adquisición a costo asequible de móviles $3 \mathrm{G}$ con acceso a Internet y a las redes sociales.

- El crecimiento de la oferta de servicio Wi-fi en instituciones educativas y lugares públicos.

- La creciente instalación de servicios de telecomunicaciones en sectores rurales.

García (2013) revela cuáles son los medios sociales líderes en el mercado peruano:

Figura 3: Medios sociales que lideran el mercado peruano.

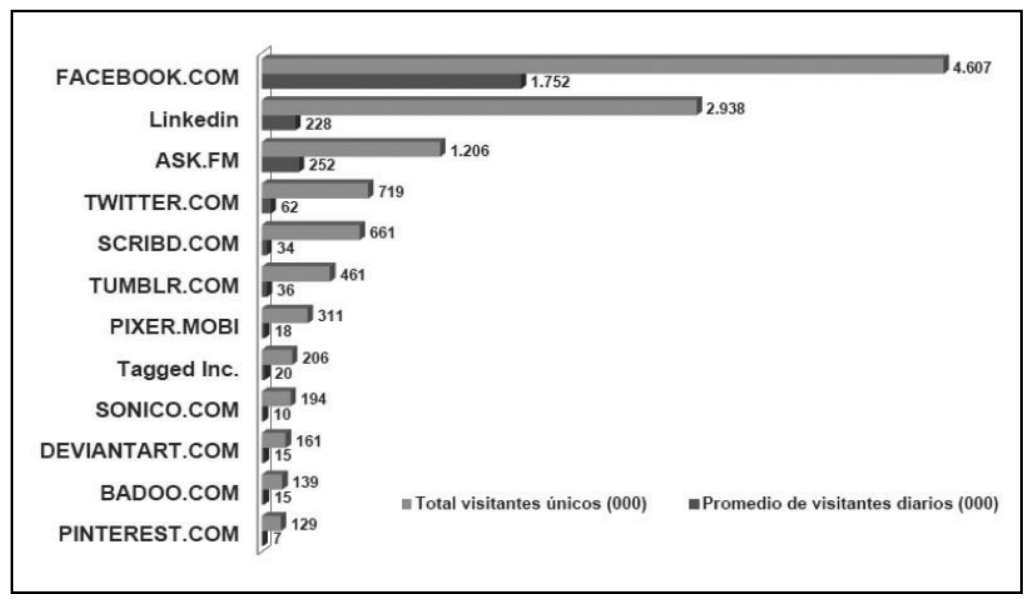

Fuente: García (2013).

\section{Los medios sociales como fuente de información}

Con el desarrollo de los medios sociales nuevas fuentes de información fueron incorporadas paulatinamente a los medios masivos, los cuales al igual que las grandes cadenas internacionales de noticias y los conglomerados de medios fueron encontrando en los medios sociales un importante recurso en la búsqueda incesante de información, que 
deviene en tarea fundamental de su actividad. Al respecto, Newman (2009: 8) estima que a partir de la crisis de las elecciones de Irán en 2009, las cadenas internacionales encontraron una serie de beneficios directos en los medios sociales.

- "Posibilidades ampliadas de acopio informativo, principalmente de fotografías y enlaces en vivo con reporteros involucrados directamente con redes.

- Una función de copia única de prueba para la actividad social de la web, reduciendo el tiempo en la organización y reduciendo el margen de errores.

- La acumulación de crédito entre comunidades tales como Twitter, incluyendo un significativo número de retornos hacia sus sitios web o emisoras".

Los periodistas han encontrado que los medios sociales son recursos de considerable importancia. En este sentido Gutiérrez y Zurita (2012) afirman que entre las actividades básicas que desarrollan los periodistas con los medios sociales las más importantes son:

- Búsqueda de noticias.

- Búsqueda de fuentes.

- Contraste de información.

Al comienzo, Twitter parecía un recurso muy ajeno al ejercicio de la actividad periodística. Se pensaba que 140 palabras no podrían decir aparentemente nada significativo que respondiera a los tradicionales estándares periodísticos y los tweets eran percibidos como un ejercicio de expresión libre sin mayor trascendencia, pero el tiempo demostró que las cosas no eran necesariamente así.

Los informativos televisivos locales usan ahora Twitter con frecuencia para diversos propósitos, algunos de los cuales describiremos en este trabajo. Un solo mensaje en Twitter difundido por los medios masivos puede tener un impacto considerable. Así, podemos recordar tweets que figuraron en las primeras planas de los diarios así como en los titulares de los informativos de TV y otros que fueron tema de la agenda pública por varios días. El 6 de diciembre de 2012 Barack Obama (@BarackObama) envió el tweet "Four more years", que alcanzó 810,000 retweets, Una hora después de que el gobierno de Sudáfrica anunciara la muerte de Nelson Mandela se habían generado más de 2 millones de tweets. El selfie de Ellen DeGeneres twiteado en plena ceremonia de entrega del Óscar (2/III/2014), ya había superado los 3 millones de retweets un día después. Todos estos casos fueron motivo de amplios comentarios en informativos de televisión y radio, así como en los diarios y revistas del mundo entero. 
Naturalmente no basta que cualquier usuario común y corriente de Twitter redacte un tweet para que de inmediato se logre un efecto de considerable magnitud. Estimamos que su trascendencia no es necesariamente la misma en todos los casos, pues depende de los siguientes factores:

- Actor social: Que por una razón u otra sea un personaje socialmente representativo y que cuente con un importante número de seguidores.

- Contexto: Sus peculiaridades de índole social y cultural influyen en la significatividad de un hecho. Lo que en un contexto merezca considerable interés tal vez en otro no determine mayor atención.

- Coyuntura: ¿Qué está pasando en el momento específico en que se envía el tweet? Aquello que en determinadas circunstancias puede pasar casi desapercibido en otro puede desencadenar un impacto mayor.

- Forma del mensaje: ¿Cómo se ha redactado? ¿Cuál es el estilo narrativo que posee? ¿Qué tanto llama la atención?

- Contenido del mensaje: El asunto del cual trata y el enfoque específico que se le ha dado.

La credibilidad es un asunto sustancial cuando se trata de reconocer la confianza que se tiene en las fuentes de información. Morris y otros (2012) realizaron un experimento vía online para conocer el grado de credibilidad de los tweets. Encontraron que Twitter es utilizado como una fuente de noticias e información, pero paradójicamente los usuarios juzgan como más creíbles los datos cuando provienen de personas que no conocen.

Cuando se habla de los temas que presentan los medios, frecuentemente nos viene a la mente la teoría del establecimiento de la agenda que, en pocas palabras, supone que los medios formulan ciertos temas que serán motivo de tratamiento informativo en determinado lapso y que en consonancia con ello se configura una agenda de discusión pública que en líneas generales tiene una relación de dependencia con lo que los medios presentan.

En nuestra época, ¿cuál es el papel de los medios sociales en la configuración de la agenda de los medios? La respuesta a esta pregunta parte del reconocimiento que el criterio original debe ser modificado, dado que los medios sociales y los propios integrantes de las redes pueden influir en la agenda mediática, en la medida en que resultan valiosas fuentes de información, tal como intentamos expresarlo en el modelo siguiente (figura 4), donde P es una persona que utiliza medios sociales. 
Figura 4: La agenda mediática en la era de los medios sociales.

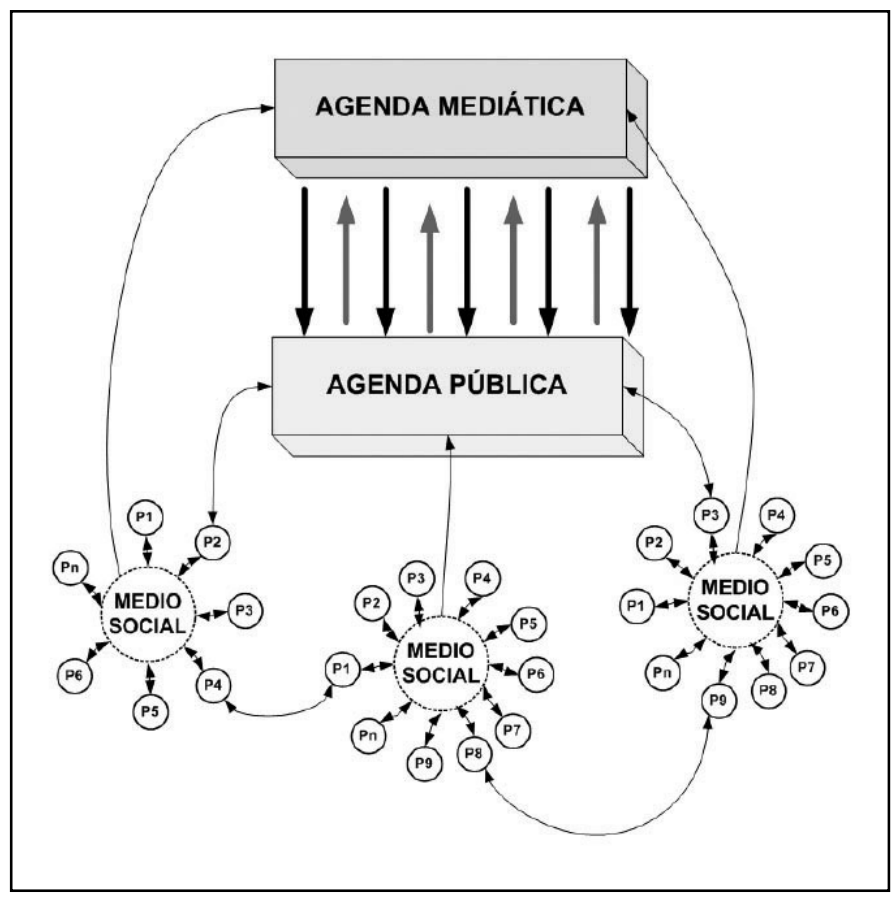

Fuente: Elaboración propia.

¿Qué periodista podría imaginarse tiempo atrás que bajo determinadas condiciones los primeros datos acerca de cierto hecho no serían alcanzados por un audaz reportero que por suerte se encontraba cerca del lugar de los hechos? Actualmente, es bastante frecuente, sobre todo durante situaciones de crisis o catástrofes, que noticias, fotos y testimonios (en audio o video) provengan de ciudadanos testigos que emplean los medios sociales gracias a sus teléfonos móviles.

En esta situación, cabe preguntarse: ¿En qué medida los periodistas hacen uso de las redes sociales para construir sus discursos? La versión más reciente de Oriella PR Network (2012) abarcó una muestra de 613 periodistas de 16 países. Encontró que más de la mitad de los periodistas entrevistados cuando buscan ideas o nuevos ángulos para redactar sus historias observan posts de páginas conocidas.

YouTube se ha convertido en una fuente de información muy importante para la televisión. Innumerables programas del mundo entero emplean el material de la red como archivo de base ya sea para informar o recrear cotidianamente. La información que puede encontrarse en este medio social es prácticamente inagotable pues cada minuto se suben 100 horas de videos, cada día el sistema de Content ID analiza más de 400 años de videos y se cuenta con más de 25 millones de archivos de referencia (YouTube, 2014). 


\section{Los medios sociales en la producción de la información}

Desde el momento en que se produce un hecho noticioso hasta su presentación a la audiencias a través de los medios masivos se atraviesan una serie de etapas de procesamiento de la información que han sido analizadas por una serie de autores y que hemos descrito recientemente (La Rosa, 2013). En pocas palabras, tomando como insumos a los materiales informativos que proceden de la realidad, son transformados para convertirse en la realidad mediática.

Estamos de acuerdo con De Maeyer (2009) cuando sostiene de modo categórico que los medios sociales determinan efectos sobre las prácticas y las rutinas cotidianas del periodismo. Para constatar este criterio basta con acercarnos a una sala de redacción actual donde Twitter, Facebook, Tumblr, YouTube, Instagram, Flickr y otros medios sociales son parte del entorno de trabajo de los periodistas. Si luego nos detenemos a revisar su producción notaremos de qué manera son utilizados estos recursos para la labor cotidiana en las salas de redacción y/o la cobertura noticiosa desde el lugar de los hechos.

Según Pignard-Cheynel y Mercier (2011) los medios sociales han acelerado los tiempos de la producción y distribución de los productos periodísticos, haciéndose efectiva una reducción en el lapso de tiempo entre la ocurrencia de los hechos y su difusión. A su entender esto se hace evidente en cuatro aspectos:

- "La desintermediación porque los ciudadanos pueden convertirse en fuentes y difusores de información que a su vez son utilizados por los medios.

- Las redes actúan como multiplicadores de las posibilidades de propagación de la información que se va difundiendo rápidamente en el ciberespacio.

- Se quiebran las barreras entre la elaboración, la difusión y la propagación de la información que se trasmite en tiempo real.

- Se alargan los tradicionales circuitos de trasmisión de la información con etapas suplementarias que no están necesariamente bajo el control de los medios".

En consonancia con el criterio previo, Levy (2010) comenta que los medios sociales son instrumentos de presión para los medios masivos que han transformado sus ciclos informativos para actualizarse constantemente. No es posible para los diarios que tienen un ciclo establecido de 24 horas pero sí resulta factible para sus plataformas en Internet que pueden irse alimentando y actualizando constantemente. Presenta como emblemáticos dos casos particularmente representativos ocurridos en el 2009:

- La primera información sobre el aterrizaje de un avión de US Airways en el Río Hudson se da a conocer mediante un tweet y una foto. El autor del mensaje viajó 
inclusive en uno de los botes de salvamento, en donde seguía tweeteando mientras que la prensa realizaba la cobertura del hecho desde las orillas del río.

- $\quad$ La muerte de Michael Jackson se publica primero en el sitio TMZ y se difunde vía Twitter antes que por otros medios.

Periodistas con experiencia de trabajo online han cubierto, de manera apropiada con sus tweets, eventos que de otra manera estaban vetados para su trasmisión en directo, tal como ocurre en el caso de la información sobre los casos que se ventilan en los juzgados de Estados Unidos, donde están prohibidas las cámaras. Periodistas que cubrieron, en los últimos años, movimientos sociales de gran trascendencia, informando a sus bases permanentemente mediante tweets: Así se estructuraba un relato vívido de lo que estaba ocurriendo, minuto a minuto, tanto más por cuanto incluían tomas fotográficas o en video.

Por ejemplo Media, Reputation and Intangibles (MRI) de la Universidad de Navarra analizó la cobertura en Twitter del atentado terrorista producido durante la maratón de Boston (15/IV/2013). The Boston Globe publicó el primer tweet a la 13:57 horas: "Un testigo informa haber escuchado dos explosiones de bombas cerca a la meta de la Maratón de Boston". 27 minutos después se publicaba la primera foto y como estrategia periodística se decidió la intensa cobertura en vivo a través de Twitter, publicando en las dos horas siguientes 150 tweets sobre el hecho. El medio adoptó una decisión riesgosa, pero efectiva, pues en cinco días logró incrementar sus seguidores en $300 \%$, demostrando que es posible el periodismo de medios sociales (Pujol, 2013).

Los diarios no pueden estar a la zaga en cuanto al uso de los medios sociales, en especial en sus ediciones virtuales, las cuales, tal como mencionamos, deben actualizarse con frecuencia. Desde mediados de 2013, muchos reporteros de diarios limeños cuentan con teléfonos inteligentes de última generación para que enviaran a la redacción central sus avances de información mientras se iban sucediendo los hechos. El material registrado podría servir para preparar una línea del tiempo o una infografía describiendo lo ocurrido, pero en todo caso resulta funcional en tanto hace posible alimentar constantemente la página web del medio. Posteriormente, sobre la base de estos datos, podría redactarse una nota más detallada para la versión impresa del diario.

Tal como lo hemos sostenido previamente, los medios sociales son importantes como cualquier otro recurso tecnológico, pero sin la intervención del profesional entrenado no pueden ser utilizados para el trabajo periodístico. Se someten a un proceso de tratamiento de la información puesto que no necesariamente todos los mensajes siguen los estándares éticos de respeto al semejante, no registran la suficiente pluralidad para ser presentados o no corresponden a los criterios de noticiabilidad preestablecidos.

El conductor del programa de radio o televisión, así como el editor del sitio web, están siempre al tanto de estos criterios que permiten presentar a la audiencia aquellos tweets o posts que sirvan a los propósitos de motivar al entrevistado o aportar a la discusión que se está presentando, desde la línea periodística del medio y en otros para ilustrar las reacciones diversas que se producen en la audiencia o las que se supone se están presentando. 
Flores (2009: 78) opina que las tecnologías amén de las formas del periodismo afectan los contenidos. En este contexto estima que el periodista debe ser:

- "Participativo: Revisar los comentarios que van ingresando y escribir sobre los que ameriten respuesta.

- Proactivo: Buscar retroalimentación antes de publicar. Configurar recursos para conocer opiniones de la audiencia antes de tratar determinado tema".

Pero como lo dice un antiguo adagio, "no todo lo que brilla es oro", de modo tal que no todo lo que se recibe a través de las redes sociales es noticiable. Kaul (2013) estima que en la era digital el periodismo debe cumplir el rol de filtrado de lo que denomina tsunami de información, aludiendo al ingente (y podríamos agregar incontrolable) volumen de datos a los cuales se tiene acceso. Esta tarea supone abordar la clasificación, selección, edición, agregado y vinculación de un conjunto de elementos ligados con las personas, las ideas y los eventos (figura 5). Será en todo caso el periodista calificado quien posee las competencias indispensables para llevar a caso estos procesos de manera eficiente en los tiempos que demanda la naturaleza del medio.

Figura 5: El "tsunami" de información y el papel del periodista.

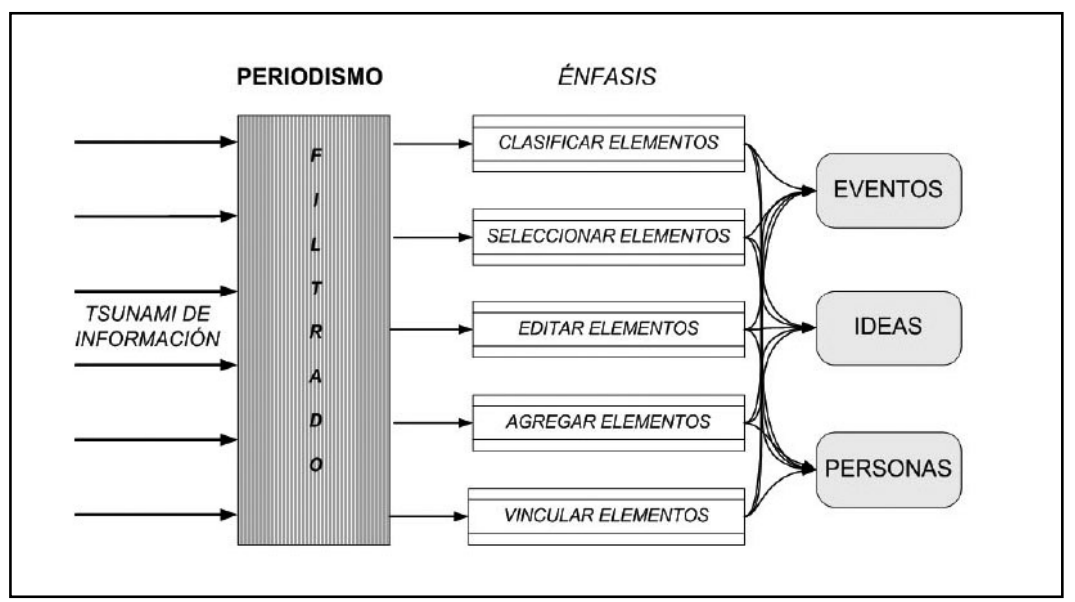

Fuente: Elaboración propia, basada en Kaul (2013).

Ante esta sobrecarga de información, Guerrini (2013: 4) propone la participación del curador de contenidos: "una persona quien selecciona la mejor información online encontrada, considerando su calidad y relevancia, la agrega y la linkea a la fuente original de la noticia, proporcionando contexto y análisis". Actuaría en este sentido como un editor de noticias, que no se centra solamente en el material específico con que cuenta sino que busca contextualizarlo y preservarlo. 
Para la verificación de los materiales se utilizan tanto recursos online como el criterio técnico del profesional que proviene del prolongado recorrido histórico de la profesión como de la propia experiencia individual. En el caso de la información audiovisual publicada en Internet y en los medios sociales existen en este sentido cuatro elementos básicos por revisar y confirmar.

- “Procedencia: ¿Es este el contenido original?

- Fuente: ¿Quién subió el contenido?

- Fecha: ¿Cuándo se creó el contenido?

- Ubicación: ¿Dónde se creó el contenido?” (Wardle, 2014: 26).

Sin embargo, cabría anotar una atingencia referida a la pluralidad del contenido de este gran volumen de información. Smymaios, Maty y Rebillard (2010) anotan que existen una gran cantidad de info-intermediarios que distribuyen elevados volúmenes de información online, la cual parece ser altamente redundante pues tiende a concentrarse en determinados asuntos. Por cierto, este cambio (en los procesos de producción) es un rasgo general observable en el periodismo digital. De acuerdo con Machado (2000: 21-22), el periodismo digital comparado con el periodismo convencional supone una reestructuración de:

- Los procesos de producción y los sistemas de circulación de la noticia.

- $\quad$ Los formatos del lenguaje.

- Las relaciones de los periodistas con los usuarios de las redes.

Ju (2012) luego de analizar los 74 diarios más importantes de los Estados Unidos asume que sigue siendo cuestionable la efectividad de Facebook y Twitter como plataformas de comunicación por cuanto encuentra que hay una distancia enorme entre el volumen de lectores y quienes se adscriben a las cuentas de medios sociales de los diarios.

No todos los usuarios de medios sociales están interesados en las noticias y hasta donde conocemos, no hay evidencia que los hipervínculos existentes en los medios sociales impliquen un incremento en el tráfico del sitio web del medio. No obstante concordamos con Lohner (2012) en la idea de que estos recursos están modificando las maneras de recibir y compartir información. Las personas se enteran muchas veces de las cosas a través de los medios sociales, pero también interactúan con el proceso noticioso al postear criterios referidos a las novedades que están conociendo.

Bastos y Zago (2013) investigaron el impacto de la lectoría de los social media en el perfil editorial de los diarios. Analizaron 2'842,699 tweets con enlaces a artículos noticiosos en los ocho diarios más importantes de Estados Unidos, Brasil, España, Reino Unido y Alemania. Comprobaron marcados cambios en la elaboración de noticias. 
Mayock (2012) realiza un análisis de los canales de retroalimentación usados por diarios online. Realizó 26 entrevistas a profundidad a editores que laboran en sitios web de diarios locales, para conocer cómo influyen en su agenda los contenidos generados por los usuarios. Encontró que los tres principales recursos empleados son Twitter, los comentarios de los lectores y Facebook.

Yezers'ka (2012) analiza los recursos interactivos en 20 ediciones digitales de diarios peruanos. Diez diarios son de circulación nacional y diez regionales. En su análisis exploratorio, la investigadora describe el acceso creciente a Internet como uno de los factores que favorece la lectura de los diarios online. En general observa un incremento en el uso de recursos interactivos. Revela que las redes sociales son usadas por 7 de cada diez diarios. El 70\% mantiene una cuenta en Facebook, el 65\% en Twitter y el 15\% un canal en YouTube. La mitad de los ciberdiarios permite usar marcadores como Google Bookmarks y Delicious.

Asimismo, la mitad atiende la opinión de sus lectores sobre las noticias preferidas y ofrece servicios RSS. Es posible comentar las noticias en el 40\% de los diarios, pero sólo el 25\% modera estos contenidos. Solo tres ciberdiarios tienen blogs de sus periodistas y 2 conceden espacios para UGC (contenidos generados por los usuarios). Además, los medios nacionales usan el $85 \%$ de los recursos interactivos evaluados, mientras que sólo lo hace el $45 \%$ de los regionales. Existen diarios que aún están en la era 1.0 y no aprovechan los recursos de Internet.

Pero ciertamente los cambios que se han producido no solo comprenden al periodista. Para cerrar este acápite nos parece conveniente consignar el aporte de Sorrentino (2013) quien asume que en el nuevo escenario periodístico contextualizado por la presencia activa de los medios sociales, se redefine la negociación entre todos los actores involucrados que participan activamente:

- 'Las fuentes realizan una constante alimentación de datos en el ambiente comunicativo en consonancia con sus intereses.

- Los periodistas trabajan con insumos (informaciones) que crecen continuamente.

- La audiencia procesa la información y la conduce a nuevos circuitos”.

\section{Los periodistas y los medios sociales}

El análisis previo nos conduce a preguntarnos cómo se relaciona el periodista con los medios sociales y con su audiencia. Para Quigley (2012) los medios sociales proporcionan una serie de beneficios a los periodistas:

- “Son instrumentos de marketing profesional.

- Su buen uso puede lograr la lealtad de los consumidores de sus noticias.

- Proporcionan una manera de encontrar y cultivar buenas fuentes. 
- Su interacción abre las puertas para que las personas proporcionen información sin necesidad de solicitarla.

- Amplifican la audiencia”.

Gutiérrez y Zurita (2012), en una encuesta a periodistas de diarios de la ciudad de Concepción (Chile), encuentran que se tiene una elevada valoración del uso de las redes sociales en el trabajo cotidiano $(91 \%)$. No obstante, la investigación realizada por Herrero (2013) muestra resultados contradictorios en una muestra de 400 periodistas españoles: el 93\% las considera una herramienta periodística, el $36.4 \%$ las utiliza para contrastar información y el $63 \%$ las considera menos confiables que los medios tradicionales.

López-Hermida y Claro (2011) en su estudio sobre los periodistas chilenos en Twitter destaca que se utiliza este medio social para contactarse con los seguidores, entablando conversaciones de naturaleza coloquial antes que para reportear o informar. Recuero (2011) estima que se hace efectiva en la práctica una interacción entre los medios periodísticos y los medios sociales. Investiga dos casos ocurridos en la misma semana de julio de 2011: la muerte de la cantante Amy Winehouse y los atentados en Oslo. El mapeo de los tweets y los retweets referidos a estas noticias le permite revelar que pese al gran volumen de información, las cuentas productoras de tweets son realmente limitadas y plantear algunas consideraciones sobre el papel del periodismo y de los medios periodísticos en el espacio de las redes sociales que presentamos en la figura 6:

Figura 6: Papel de los medios periodísticos en el espacio de las redes sociales.

\begin{tabular}{|l|l|}
\hline \multicolumn{1}{|c|}{$\begin{array}{c}\text { PAPEL DE MEDIOS } \\
\text { PERIODÍSTICOS }\end{array}$} & DESCRIPCIÓN \\
\hline Legitimación & Cuando la información es publicada por un medio periodístico. \\
\hline Credibilidad & $\bullet \quad$ Tweets y retweets refuerzan credibilidad de los medios. \\
\hline Filtro & $\bullet \quad$ Se concede a los actores que citan medios periodísticos. \\
\hline Jerarquización & Los medios filtran las informaciones relevantes. \\
\hline
\end{tabular}

Fuente: Recuero (2011).

Por su parte Sozeri (2011) categoriza los tweets de los periodistas según su contenido:

- “Agenda: Los tweets analizan el contenido noticioso de los medios principales sin colocar hiperpervínculos.

- $\quad$ Ligados al propio medio. 
- Ligados a otros medios: Referidos a medios con los cuales no trabaja.

- Menciones a periodistas: Los que se refieren a sus colegas o amigos.

- Menciones a otros: Que se refieren a personas que no son periodistas o a la audiencia.

- Retweets: Difunden los tweets de otros.

- Actividades cotidianas: Se refieren a sus labores de cada día".

Aun cuando siempre se ha planteado como supuesta una vinculación permanente, tradicionalmente ha existido cierta distancia entre el periodista y la audiencia. Inclusive ya desarrollada la web 1.0 se mantenían los correos electrónicos de los periodistas como datos reservados, al menos para la audiencia en general. No obstante con el desarrollo de la web $2.0 \mathrm{y}$ de la interactividad que supone es frecuente que se promocione la cuenta en Twitter y Facebook de los periodistas de los medios así como de los programas que conducen.

En tal sentido, el caso de la televisión aparece un scroll o un texto en pantalla con las cuentas en Facebook o Twitter de los periodistas. Tanto más, mientras Patricia Janiot presenta el programa Panorama Mundial en CNN en español, invita con cierta frecuencia a los televidentes a conectarse con ella a través de sus cuentas@patriciajaniot y https:// www.facebook.com/PatriciaJaniotCNN.

Ahora bien, usando las expresiones de redes sociales, ¿cuál es la visibilidad de los periodistas en las redes sociales?

Galant (2013) publicó la relación de los 10 periodistas estadounidenses con más seguidores en Twitter (figura 7):

Figura 7: Periodistas con más seguidores en Twitter. Incremento 2012-2013.

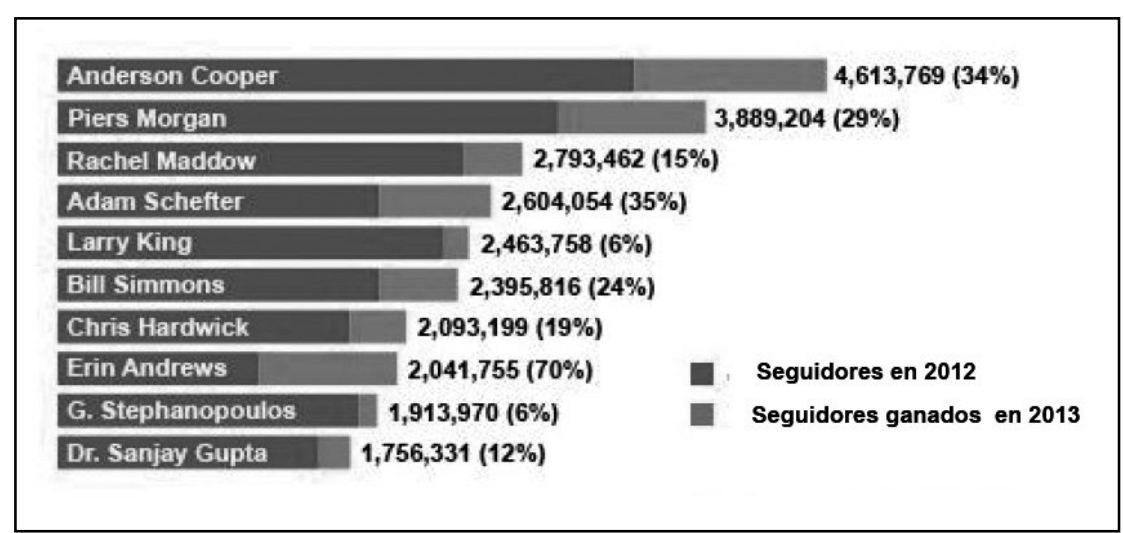

Fuente: Galant (2013). 
Iluminati Lab (2013) presenta un ranking de los periodistas latinoamericanos más populares en Twitter (figura 8):

Figura 8: Periodistas latinoamericanos más populares en Twitter.

\begin{tabular}{|c|c|r|}
\hline PAÍ́ & PERIODISTA & \multicolumn{2}{|c|}{ SEGUIDORES } \\
\hline Argentina & Jorge Rial & $2,359,316$ \\
\hline Chile & Soledad Onetto & $1,110,850$ \\
\hline Colombia & Andrea Serna & $1,737,013$ \\
\hline Ecuador & Estefani Espin & 262,234 \\
\hline México & Carlos Loret de Mola & $2,875,726$ \\
\hline Venezuela & Nelson Bocaranda & $1,508,789$ \\
\hline
\end{tabular}

Fuente: Illuminati Lab (2013).

Vox Populi (2013) revela cuáles son los 10 periodistas peruanos más populares en Facebook y Twitter (figura 9):

Figura 9: Periodistas peruanos más populares en Facebook y Twitter.

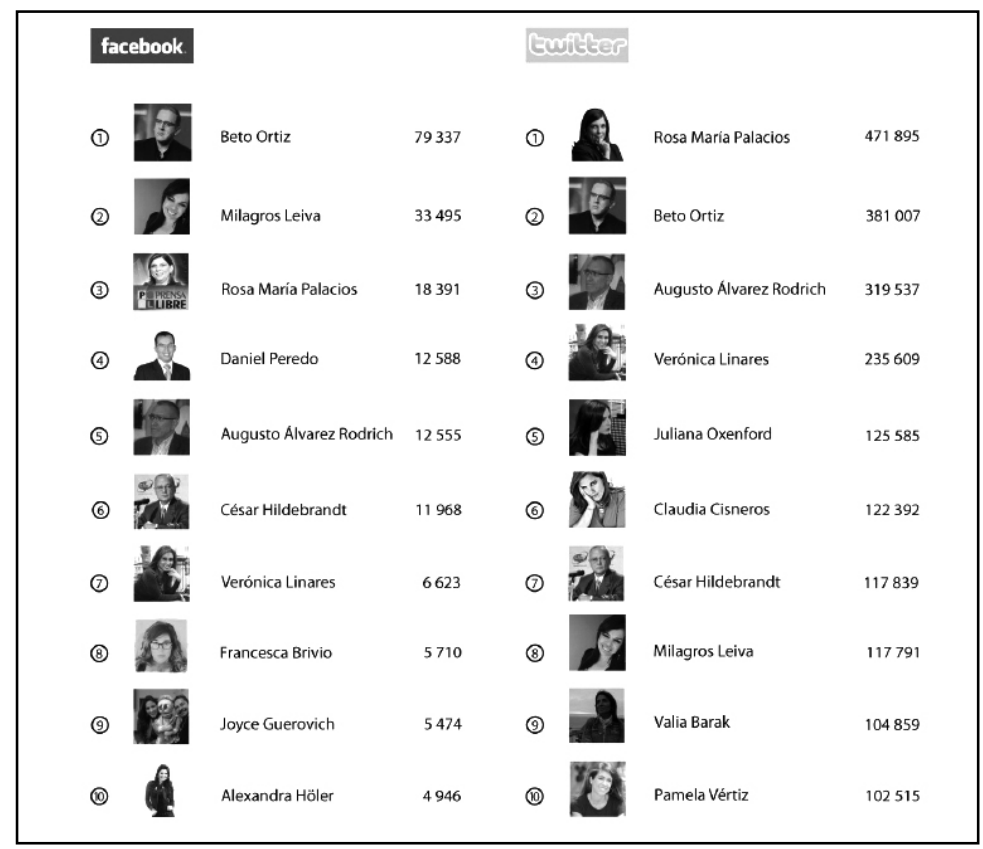

Fuente: Vox Populi (2013). 
Una rápida apreciación de los periodistas más populares en Facebook y Twitter en los entornos citados nos permite concluir que existe un marcado predominio de los periodistas quienes trabajan en televisión. Ello se debería probablemente al impacto mediático que alcanzan y a la credibilidad que suele adjudicársele.

Los medios sociales pueden ser importantes elementos de apoyo para los periodistas en situaciones difíciles. Desde el 2013, Egipto apresó a un grupo de periodistas de la cadena Al Jazeera acusándolos de estar implicados con grupos terroristas. La campaña lanzada el 1 de febrero de 2014 usando el hashtag \#FreeAJstaff alcanzó 250 millones de visitas. Asimismo, se ha utilizado la innovadora herramienta Thunderclap que permite colocar el mismo mensaje a la vez en Twitter, Facebook y Tumblr. En diversos lugares del mundo miles de periodistas salieron a las calles el 27 de febrero portando pancartas y chalecos con el hashtag y el lema "El periodismo no es un crimen" (Tenore, 2014).

\section{Reflexiones finales}

Los niveles de obsolescencia de la tecnología muy acelerados nos obligan a estar permanentemente atentos a los cambios así como a descartar muy pronto equipos y recursos de comunicación. Si bien existen medios sociales que tienen los mayores rangos de utilización a nivel internacional, están disponibles recursos de diverso orden, para uso y satisfacción de nichos diversos. Seguramente que en el futuro se desarrollarán muchos otros y tal vez desaparezcan algunos.

No sabemos a ciencia cierta qué nos traerá el futuro en el campo de los medios sociales ni cómo ello estará influenciado por el desarrollo de la tecnología $4 \mathrm{G}$ en los teléfonos móviles y de la banda ancha o por otras innovaciones. Seguramente, sofisticados programas harán posible nuevas modalidades de categorización automatizada de las clases de información de acuerdo a categorías preestablecidas y nuevas aplicaciones de los medios sociales facilitarán el trabajo cotidiano del periodista.

No obstante, por más avances que se logren en el desarrollo tecnológico, se requerirá siempre del trabajo del ser humano, del profesional en periodismo quien cuente con las competencias necesarias para encontrarle la utilidad a los recursos que se vayan inventando y sepa aplicarlos tanto para el desarrollo de la profesión en general como para servir mejor a su comunidad, que es una de las funciones esenciales que se hizo efectiva desde los albores de la actividad periodística.

Estamos convencidos que ningún estudio prospectivo podrá demostrar que es posible prescindir de los seres humanos como elementos fundamentales para el procesamiento y presentación de la información así como para la multiplicidad de decisiones que ello involucra. 


\section{Fuentes consultadas}

Alexa (2014). “The top 500 sites on the web". Extraída el 11/IV/2014 desde http://www. alexa.com/topsites

Boyd, D. (2011). "Social network sites as networked publics: Affordances, dynamics, and implications", pp. 39-58. En Papacharissi, Z. (ed.). A networked self. Identity, community and culture on social network sites. Londres: Routledge.

Boyd, D. y Ellison, N. (2007). "Social Network Sites: Definition, history and scholarship", Journal of Computer-Mediated Communication. Vol. 13, núm. 1, pp. 210-230.

Bruns, A. (2013). "From Prosumption to Produsage", pp. 67-78. En Towse, R. y Handke, C. (eds.). Handbook on the Digital Creative Economy. Gloucestershire: Edward Elgar.

Cabañas Sánchez, G. (2011). Extracción y análisis de información del servicio de red social Twitter, a través de la plataforma de "cloud computing" Google App Engine [Proyecto de fin de carrera]. Madrid: Universidad Carlos III. Extraída el 29/IV/2014 desde http:/earchivo.uc3m.es/handle/10016/12973

De Maeyer, J. (2009). "Journalism practices in an online environment. An investigation into the evolving specificities of newswork in Belgium, as challenged by technological changes". Extraída el 29/IV/2014 desde http://juliettedm.files.wordpress.com/2010/04/ journa lism_practices.pdf

Duggan, M. y Smith, A. (2013, diciembre 30). "Social Media Update 2013”. Extraída el 20/IV/2014 desde http://www.pewinternet.org/2013/12/30/social-media-update-2013/

Flores Vivar, J. (2009). "Nuevos modelos de comunicación, perfiles y tendencias en las redes sociales". Comunicar, núm. 33, pp. 73-81. Extraída el 29/IV/2014 desde http://www. redalyc.org/pdf/158/15812486009.pdf

Galant, G. (2013). “2013: A year in Twitter Journalism”. Extraída el 29/IV/2014 desde http://muckrack.com/daily/2013/12/30/2013-a-year-in-twitter-journalism/

García, M. (2013, septiembre 12). "Futuro Digital Perú 2013”. Extraída el 29/IV/2014 desde http://www.comscore.com/lat/Insights/Presentations_and_Whitepapers/2013/2013 Peru_Digital_Future_in_Focus

García Govaze, D. (2011). Cambio de paradigm en Internet. Análisis y tendencias del Social Media [Projecte final de carrera en Enginyeria de Telecomunicació]. Barcelona: Universitat Técnica de Catalunya, Escola Tècnica Superior d' Enginyeria de Telecomunicació de Barcelona.

Guerrini, F. (2013). Newsrooom Curators and Independent Storytellers: Content curation 
as a New Form of Journalism. Oxford: Reuters Institute for the study of Journalism, University of Oxford

Gutiérrez, F. y Zurita, L. (2012). "Las redes sociales y su impacto en el proceso de preparación y producción de noticias: Análisis de las rutinas de los periodistas a partir de tres diarios chilenos”. Comuni@cción. Vol. III, núm. 1, pp.36-44.

Herrero, E. (2013). Periodistas y redes sociales en España: Del 11 M al 15 M (2004-2011) [tesis doctoral]. Madrid: Universidad Carlos III de Madrid, Departamento de Periodismo y Comunicación Audiovisual.

Illuminati Lab (2013, julio 25). “¿Quiénes son los periodistas latinoamericanos más populares en Twitter?”. Extraída el 29/IV/2014 desde http://illuminatilab.com/blog/ uncategorized/quienes-son-los-periodistas-latinoamericanos-mas-populares-en-twitter/

ITU (2013). “The World in 2013: ICT Facts and Figures”. Extraída el 20/IV/2014 desde http://www.itu.int/en/ITU-D/Statistics/Documents/facts/ICTFactsFigures2013-s.pdf

Ju, A. (2010). Evaluating the Effectiveness of Facebook and Twitter as New Publishing Platforms for Newspapers [tesis de maestría]. Austin: University of Texas, Graduate School.

Kaul, V. (2013). "Journalism in the age of digital technology". Online Journal of Communication and media technologies. Vol. 3, núm. 1, pp. 125-143. Extraída el 29/ IV/2014 desde http://www.ojcmt.net/articles/31/317.pdf

La Rosa, A. (2013). Construcción de la agenda mediática: Una mirada al interior de la Comunicación. Lima: Universidad de San Martín de Porres.

Levy, G. (2010). Social Media and Journalism: How the Internet and Social Networking Have Changed Journalism's Workflow [tesis]. Massachusetts: Tufts University. Extraída el 29/IV/2014 desde http://repository01.lib.tufts.edu:8080/fedora/get/ tufts:UA005.005.065.00001/ bdef:TuftsPDF/getPDF

Lohner, J. (2012). Facebook, Twitter, and interactivity: Changes in the ways society discusses news [tesis de grado]. Ohio: Ohio University.

López-Hermida, A. y Claro, C. (2011). "Medios y periodistas en Twitter: El caso chileno". Correspondencia y Análisis, núm. 1, pp. 17-33.

Machado, E. (2000). La estructura de la noticia en las redes digitales: Un estudio de las consecuencias de las metamorfosis tecnológicas en el periodismo [Tesis doctoral]. Barcelona: Universidad Autónoma de Barcelona, Facultad de Ciencias de la Comunicación. 
Mayock, P. (2012). Agenda Building in the Age of Online Audience Feedback [tesis de maestría]. Ohio: Kent State University, College of Communication and Information.

Morris, M. y otros (2012). "Twetting is believing? Understanding microblog credibility perceptions". Extraída el 29/IV/2014 desde http:/www.social4retail.com/uploads/ $1 / 0 / 9 / 8 / 10981970 /$ tweet_credibility_study.pdf

Newman, N.

(2011). "Mainstream media and the distribution of news in the age of social discovery". Extraída el 29/IV/2014 desde https://reutersinstitute.politics.ox.ac.uk/fileadmin/documents/ Publications/Working_Papers/Mainstream_media_and_the_distribution_of_news_.pdf (2009). "The rise of social media and its impact on mainstream journalism”. Extraída el 29/ IV/2014 desde https://reutersinstitute.politics.ox.ac.uk/fileadmin/documents/Publications/ The_rise_of_social_media_and_its_impact_on_mainstream_journalism.pdf

Oriella PR Network (2012). "The influence game: How news is sourced and managed today. Global Digital Journalism Study 2012”. Extraída el 29/IV/2014 desde http://www. oriellaprnetwork.com/sites/default/files/research/Oriella\%20Digital\%20Journalism\%20 Study\%202012\%20Final\%20US.pdf

Pignard-Cheynel, N. y Mercier, A. (2011). "L'appropriation des réseaux sociaux par les webjournalistes en France". Extraída el 29/IV/2014 desde http://www.medias011.univcezanne.fr/fileadmin/Medias11/Documents/A4/MERCIER_PICHARD.pdf

Pujol, F. (2013). "Social Media Journalism. Twitter Live Coverage of Crisis - Risks and Rewards for@bostonglobe - Impact Analysis Boston Marathon Bombing - April 2013". Extraída el 19/IV/2014 desde http://reputation-metrics.org/2013/04/19/social-mediajournalism-twitter-live-coverage-of-crisis-risks-and-rewards-for-bostonglobe-impactanalysis-boston-marathon-bombing-april-2013/

Quigley, R. (2012). "Lesson 2: Interaction. Course Social media for journalists". Austin: University of Texas at Austin, Knight Center.

Recuero, R. (2011). “'Deu no Twitter, alguém confirma?’ Funções do Jornalismo na Era das Redes Sociais". Ponencia presentada en el $9^{\circ}$ Encontro Nacional de Pesquisadores em Jornalismo. Rio de Janeiro.

Smymaios, M.; Marty, E. y Rebillard, F. (2010). "Does the Long Tal apply to online news? A quantitative study of French-speaking news websites". New Media \& Society. Vol. 12, núm. 8 , pp. 1244-1261.

Sorrentino, C. (2013). "El equívoco del periodismo ciudadano. Cómo las nuevas formas de consumo de la información están redefiniendo el periodismo". adComunica. Revista de Estrategias, Tendencias e Innovación en Comunicación, núm. 6, pp. 21-37. 
Sozeri, C. (2011). "Does social media reduce 'corporate media influence' on journalism? The Case of Turkish Media”. Estudos em Comunicação, núm. 10, pp. 71-92.

The cocktail analysis (2011, febrero 21). "Informe de resultados. Observatorio Redes Sociales $3^{\circ}$ oleada". Extraída el 29/IV/2014 desde http://www.slideshare.net/TCAnalysis/ tca-observatorio-redes-sociales2011publico

Tenore, M. (2014, febrero 27). "People around the world show support for Al Jazeera journalists". Extraída el 29/IV/2014 desde http://ivoh.org/people-around-world-showsupport-al-jazeera-journalists/

Toledo Bastos, M. y Zago, G. (2013). "Tweeting News Articles. Readership and News Sections in Europe and the Americas". Extraída el 29/IV/2014 desde http://classic.sgo. sagepub.com/content/3/3/2158244013502496

Vox Populi (2013). "Barómetro 1 Ranking de Redes Sociales en Perú, Junio". Extraída el 20/X/2013 desde http://www.voxpopuli.pe/pdf/2013-06\%20brrsp.pdf/

Wardle, C. (2014). "Verifying user-generated content", pp. 24-33. En Silverman, C. Verification handbook; An ultimate guideline on digital age sourcing for emergency coverage. Maastricht: European Journalism Center.

Yezers'ka, L. (2012). "Gestión de los recursos informativos en los ciberdiarios peruanos". Comunifé, núm. 12, pp. 12-31.

YouTube (2014). “Estadísticas”. Extraída el 29/IV/2014 desde https://www.youtube.com/ $\mathrm{yt} / \mathrm{press} / \mathrm{es} / \mathrm{statistics.html}$ 